\title{
CORRELATION BETWEEN PERCEIVED SOCIAL SUPPORT AND LONELINESS AMONG CHINESE ADOLESCENTS: MEDIATING EFFECTS OF PSYCHOLOGICAL CAPITAL
}

\author{
Yujia $\operatorname{Ren}^{1} \&$ Binbin $\mathbf{J i}^{2}$ \\ ${ }^{1}$ Physical Education Institute, Hunan First Normal University, Changsha, 410205, China \\ ${ }^{2}$ School of Nursing, Hunan University of Chinese Medicine, Changsha, 410205, China
}

received: 22.10.2018;

revised: 25.5.2019;

accepted: 3.6 .2019

SUMMARY

Background: Adolescence is a period characterized by high frequency of mental health problems. Loneliness, in particular, is a common psychological problem. This study aims to explore the effects of psychological capital on adolescents' loneliness and the mediating effects of perceived social support.

Subjects and methods: A total of 694 adolescents from six regions in Hunan Province, China were selected. The survey was conducted using the Perceived Social Support Scale, Loneliness Scale, and Psychological Capital Questionnaire. The study analyzed the correlations between perceived social support, psychological capital, and loneliness among adolescents and the mediating role of psychological capital in the relationship between perceived social support and loneliness.

Results: Significant correlations were observed between adolescents' total scores in perceived social support, psychological capital, and loneliness and between each dimension. The following observations were made: a significant negative correlation between perceived social support and loneliness $(r=0.440, P<0.01)$, a significant positive correlation between perceived social support and psychological capital $(r=0.493, P<0.01)$, and a significant negative correlation between psychological capital and loneliness $(r=-0.303, P<0.01)$. Psychological capital plays a statistically significant mediating role on the relationship between perceived social support and loneliness $(P<0.01)$.

Conclusions: Perceived social support and psychological capital can reduce adolescents' loneliness and perceived social support can alleviate loneliness by enhancing psychological capital.

Key words: loneliness - perceived social support - psychological capital - adolescents

$$
* * * * *
$$

\section{INTRODUCTION}

In the development of an individual's life, adolescence is considered to be the period when one is most prone to emotional and behavioral problems. Loneliness is one of the typical emotional problems that individuals encounter during adolescence (Cavanaugh \& Buehler 2016). Loneliness is a diffused painful inner experience that occurs when an individual faces an obviously insufficient social relationship, either qualitatively or quantitatively. This painful experience is accompanied frequently by significant features, such as "no sense of value, emptiness, loss of self-control and personal threats" (Mosher et al. 2012, Holt-Lunstad et al. 2015). Previous studies have shown that loneliness is typically associated closely with depression and separation, whereas depression and separation reduce adolescents' need for belonging and self-esteem (He et al. 2014). Loneliness may also lead to anxiety, alcohol addiction, and even suicide (Heinrich \& Gullone 2006, Jaremka et al. 2013). Hence, the study on adolescents' loneliness has become one of the key research topics in the field of education and development psychology in recent years.

Among the various factors that affect adolescents' loneliness, the role of perceived social support has attracted considerable attention from researchers (Liu et al. 2016). Perceived social support refers to the level of satisfaction an individual feels with regard to the respect, understanding, and support that they perceive in their living environment (Ye et al. 2014). For adolescents, their main social support comes from their parents, that is, the family system. However, peer relationship becomes an increasingly important source of social support with the increase in age and shift in life focus (Laird et al. 2016). Social support plays an important buffering role in adolescents' social adaptation and can reduce the incidence of mental illness, such as loneliness (Park et al. 2013).

Apart from perceived social support, which has a positive effect on loneliness, psychological capital is another important factor that has gained the attention of researchers. Psychological capital refers to a positive psychological state manifested in individuals in the process of growth and development and is a psychological resource for promoting personal growth (Peterson et al. 2011). At present, the theory of psychological capital has become a focal point in the field of positive psychology. Previous studies have shown that psychological capital includes psychological resilience, hope, optimism, and many other positive psychological qualities conducive to the healthy development of an individual's body and mind (Carmona-Halty et al. 2019). Attention has also been given to the relationship between psychological capital and loneliness. Psychological capital is an important protective factor that reduces loneliness (Bian \& Leung 2015, Fan et al. 2017). 
Studies on the relationship between perceived social support and psychological capital, between perceived social support and loneliness, and between psychological capital and loneliness have been conducted. However, no research has linked perceived social support with psychological capital and loneliness and explored the internal working processes and mechanism. Based on previous achievements, this paper intends to fill this research gap by examining comprehensively the relationship among perceived social support, psychological capital, and loneliness. The present paper also applied the theory of mediating effect to explore the mediating role of psychological capital on the relationship between social support and loneliness. The paper aims to provide theoretical guidance for preventing and alleviating effectively the sense of loneliness among adolescents and improving their level of perceived social support.

\section{SUBJECTS AND METHODS}

\section{Participants}

This study was conducted from September 2018 to October 2018. For this study, 694 adolescents were selected from six regions (Changsha, Liuyang, Shuanfeng, Yueyang, Shaoyang, and Chenzhou) in Hunan Province, China using the random cluster sampling method. A total of 364 males and 330 females participated in the questionnaire survey. The ages of the respondents ranged from 12 to 15 years with an average of $13.97 \pm 0.85$.

\section{Instruments}

(1) The Perceived Social Support Scale (Zimet et al. 1990) consists of 12 items, each of which includes seven grades that range from 12 to 84 points. The three subscales measured are friend support, family support, and other perceived support. Each subscale contains four items. Yan \& Zheng (2006) made modifications on this scale and changed "colleagues, relatives, and leaders" to "classmates, relatives, and teachers". The sum of the scores from the three subscales is the total score of the Perceived Social Support Scale. A high total score indicates the total level of social support as perceived by the individual is high.

(2) The Psychological Capital Questionnaire as compiled by Fan et al. (2015) was also used. The questionnaire is divided into five dimensions, namely, selfreliance and tenacity, optimism and openness, tolerance and friendliness, reasoning and gratitude, and selfconfidence and progress-making. Each dimension consists of five items for a total of 25 items. The scale is scored using a five-point Likert scale ranging from " 1 = completely consistent" to " $5=$ completely inconsistent". The dimension score is calculated after the answers for the forward-represented items are reverse-converted. A high score indicates a corresponding high psychological capital level. Cronbach's $\alpha$ coefficient of the questionnaire is 0.88 , which indicates the questionnaire has good reliability and validity and is suitable for individuals aged 16 and below.

(3) The Loneliness Scale (Zhou et al. 2012) consists of eight items, including six positive order items for "loneliness" and two reverse order items for "nonloneliness" (i.e., item 3: "I am a person willing to make friends" and item 6: "When I am sad, I can find someone to accompany me"). Each item is scored using a four-level frequency scoring from 1 (never), 2 (seldom), 3 (sometimes), and 4 (always). Positive statement items, that is, items for non-loneliness, are scored in reverse order. The total score is 8 to 32 points. A high total score means a high degree of loneliness (Zhou et al. 2012).

\section{Statistical Analysis}

Data were analyzed using SPSS 21.0 and AMOS7.0 statistical software. The quantitative data were expressed as mean \pm standard deviation. The analysis of the correlation between variables was conducted using Pearson's correlation analysis. The mediating effect was analyzed using multiple linear regression analysis with the difference statistically significant at $\mathrm{P}<0.05$.

\section{RESULTS}

\section{General data}

Table 1 shows that among the 694 adolescents, 364 were males and 330 were females. Among the respondents, 12, 228, 226, and 228 were aged 12, 13, 14, and 15 years, respectively, with an average age of $13.97 \pm 0.85$.

Table 1. General data of study object

\begin{tabular}{lcc}
\hline & $\mathrm{N}$ & Percent (\%) \\
\hline Gender & & \\
Male & 364 & 52.4 \\
Female & 330 & 47.6 \\
Years & & \\
12 & 12 & 1.7 \\
13 & 228 & 32.9 \\
14 & 226 & 32.6 \\
15 & 228 & 32.9 \\
\hline
\end{tabular}

\section{Descriptive statistics of score of each scale}

According to the statistical analysis, the adolescents' scores in the three dimensions of perceived social support, namely, friend support, family support, and other perceived support, and their total score are $19.91 \pm 5.25,19.24 \pm 5.28,19.18 \pm 5.27$, and $58.39 \pm 13.40$, respectively. The scores in the five dimensions of psychological capital, namely, self-reliance and tenacity, optimism and openness, tolerance and friendliness, reasoning and gratitude, and self-confidence and progress-making, and their total score are $3.93 \pm 0.80$, $3.72 \pm 0.72, \quad 3.73 \pm 0.91, \quad 3.40 \pm 0.74, \quad 3.74 \pm 0.75$, and $3.70 \pm 0.59$, respectively. The score for loneliness is $15.38 \pm 5.00$. Table 2 shows the results. 


\section{Correlation among perceived social support, psychological capital, and loneliness}

Tables 3-5 present the results of Pearson's correlation analysis for perceived social support, psychological capital, and loneliness, respectively. Significant correlations were observed among perceived social support, psycho logical capital, and loneliness. Specifically, the following observations were made: a significant negative correlation between perceived social support and loneliness $(\mathrm{r}=-0.440, \mathrm{P}<0.01)$, a significant positive correlation between perceived social support and psychological capital $(\mathrm{r}=0.493, \mathrm{P}<0.01)$, and a negative correlation between psychological capital and loneliness $(\mathrm{r}=-0.303, \mathrm{P}<0.01)$.

Table 2. Scores for perceived social support, psychological capital, and loneliness

\begin{tabular}{lcccc}
\hline Index & $\begin{array}{c}\text { Theoretical } \\
\text { minimum value }\end{array}$ & $\begin{array}{c}\text { Theoretical } \\
\text { maximum value }\end{array}$ & Mean & Standard deviation \\
\hline Other support & 4.00 & 28.00 & 19.91 & 5.25 \\
Family support & 4.00 & 28.00 & 19.24 & 5.28 \\
Friend support & 4.00 & 28.00 & 19.18 & 5.27 \\
Total score in perceived social support & 12.00 & 84.00 & 58.39 & 13.40 \\
Self-reliance \& tenacity & 1.00 & 5.00 & 3.93 & 0.80 \\
Reasoning \& gratitude & 1.00 & 5.00 & 3.72 & 0.72 \\
Tolerance \& friendliness & 1.00 & 5.00 & 3.73 & 0.91 \\
Self-confidence \& progress-making & 1.00 & 5.00 & 3.40 & 0.74 \\
Optimism \& openness & 1.00 & 5.00 & 3.74 & 0.75 \\
Index & 1.00 & 5.00 & 3.70 & 0.59 \\
Other support & 8.00 & 32.00 & 15.38 & 5.00 \\
\hline
\end{tabular}

Table 3. Correlation between perceived social support and loneliness

\begin{tabular}{lcccc}
\hline Index & Other support & Family support & Friend support & Perceived social support \\
\hline Other support & 1 & & & \\
Family support & $0.607^{* *}$ & 1 & & \\
Friend support & $0.704^{* *}$ & $0.442^{* *}$ & 1 & 1 \\
Perceived social support & $0.903^{* *}$ & $0.817^{* *}$ & $0.810^{* *}$ & $-0.440^{* *}$ \\
Loneliness & $-0.426^{* *}$ & $-0.346^{* *}$ & $-0.345^{* *}$ & \\
\hline
\end{tabular}

Table 4. Correlation between perceived social support and psychological capital

\begin{tabular}{|c|c|c|c|c|c|c|c|c|c|}
\hline Index & 1 & 2 & 3 & 4 & 5 & 6 & 7 & 8 & 9 \\
\hline 1 Other support & 1 & & & & & & & & \\
\hline 2 Family support & $0.607 * *$ & 1 & & & & & & & \\
\hline 3 Friend support & $0.704 * *$ & $0.442 * *$ & 1 & & & & & & \\
\hline 4 Perceived social support & $0.903 * *$ & $0.817 * *$ & $0.810^{* *}$ & 1 & & & & & \\
\hline 5 Self-reliance \& tenacity & $0.381 * *$ & $0.387 * *$ & $0.258 * *$ & $0.415^{* *}$ & 1 & & & & \\
\hline 6 Reasoning \& gratitude & $0.328 * *$ & $0.295^{* *}$ & $0.211 * *$ & $0.332 * *$ & $0.497 * *$ & 1 & & & \\
\hline 7 Tolerance \& friendliness & $0.245^{* *}$ & $0.195^{* *}$ & $0.199 * *$ & $0.256^{* *}$ & $0.362 * *$ & $0.384 * *$ & 1 & & \\
\hline 8 Self-confidence \& progress-making & $0.372 * *$ & $0.343 * *$ & $0.308^{* *}$ & $0.401 * *$ & $0.459 * *$ & $0.550 * *$ & $0.370 * *$ & 1 & \\
\hline 9 Optimism \& openness & $0.431 * *$ & $0.362 * *$ & $0.398 * *$ & $0.461 * *$ & $0.474 * *$ & $0.505 * *$ & $0.405 * *$ & $0.540 * *$ & 1 \\
\hline 10 Psychological capital & $0.465 * *$ & $0.418 * *$ & $0.364 * *$ & $0.493 * *$ & $0.744 * *$ & $0.768 * *$ & $0.701 * *$ & $0.767 * *$ & $0.772^{* *}$ \\
\hline
\end{tabular}

Table 5. Correlation between psychological capital and loneliness

\begin{tabular}{|c|c|c|c|c|c|c|}
\hline Index & 5 & 6 & 7 & 8 & 9 & 10 \\
\hline 5 Self-reliance \& tenacity & 1 & & & & & \\
\hline 6 Reasoning \& gratitude & $0.497 * *$ & 1 & & & & \\
\hline 7 Tolerance \& friendliness & $0.362 * *$ & $0.384 * *$ & 1 & & & \\
\hline 8 Self-confidence $\&$ progress-making & $0.459 * *$ & $0.550 * *$ & $0.370 * *$ & 1 & & \\
\hline 9 Optimism \& openness & $0.474 * *$ & $0.505 * *$ & $0.405 * *$ & $0.540 * *$ & 1 & \\
\hline 10 Psychological capital & $0.744 * *$ & $0.768 * *$ & $0.701 * *$ & $0.767 * *$ & $0.772 * *$ & 1 \\
\hline 11 Loneliness & $-0.208 * *$ & $-0.210 * *$ & $-0.137 * *$ & $-0.214^{* *}$ & $-0.384 * *$ & $-0.303 * *$ \\
\hline
\end{tabular}


Table 6. Mediating role of psychological capital in the relationship between perceived social support and loneliness

\begin{tabular}{llcccrc}
\hline \multirow{2}{*}{ Dependent variable } & \multirow{2}{*}{ Independent variable } & \multicolumn{2}{c}{ Non-standard coefficient } & Standard & t & P \\
& & B & Standard error & coefficient & & \\
\hline Loneliness & Constant & 24.970 & 0.763 & & 32.746 & 0.000 \\
& Perceived social support & -0.164 & 0.013 & -0.440 & -12.904 & 0.000 \\
Psychological capital & Constant & 2.443 & 0.087 & & 28.217 & 0.000 \\
& Perceived social support & 0.022 & 0.001 & 0.493 & 14.920 & 0.000 \\
Loneliness & 27.331 & 1.112 & & 24.571 & 0.000 \\
& Constant & -0.143 & 0.015 & -0.385 & -9.853 & 0.000 \\
& Perceived social support & -0.966 & 0.333 & -0.113 & -2.901 & 0.004 \\
\hline
\end{tabular}

\section{Mediating role of perceived social support}

According to the correlation analysis in Table 3-5, perceived social support is negatively correlated with loneliness; psychological capital and loneliness are negatively correlated and perceived social support and psychological capital are positively correlated, thereby providing a premise for the mediating effect test. According to the introduction of mediating effect by Wen et al. (2004), psychological capital is assumed to be a mediating variable, perceived social support an independent variable, and loneliness a dependent variable. In the first step, perceived social support was used as an independent variable and loneliness was used as a dependent variable. In the second step, perceived social support was used as an independent variable and psychological capital was used as a dependent variable. In the third step, perceived social support and psychological capital was used as independent variables, while loneliness was used as a dependent variable. Table 6 shows the analysis results of the mediating effect of psychological capital. Table 4 shows that results of the regression analysis indicates perceived social support can predict loneliness $(\mathrm{P}=0.000)$, psychological capital significantly predicts loneliness $(\mathrm{P}=0.000)$, and perceived social support significantly predicts psychological capital $(\mathrm{P}=0.000)$. The results of the third step suggest that after the appearance of psychological capital, the beta $(\beta)$ of perceived social support to loneliness changed from -0.440 to -0.385 , indicating that psychological capital plays a mediating role in their relationship. In short, the test results of the three steps are statistically significant $(\mathrm{P}<0.01)$, showing that psychological capital plays a partial mediating role in the relationship between adolescents' social support and loneliness.

\section{DISCUSSION}

\section{Analysis of the current situation of perceived social support, psychological capital and loneliness among adolescents}

According to the results of this study, adolescents' total score in social support is approximately 20 points. The scores in family support, friend support, and other types of support are all around 19 points. These results differ from those of the survey on 635 middle school students by Zhang et al. (2017). In the study by Fan et al. (2017), adolescents' scores in the three dimensions of perceived social support are all around 22 points, significantly higher than the results of this study. Previous studies have pointed out that adolescents' perception of external social support is often influenced by their inner conflicts. Despite the increase of objective social support during adolescence, adolescents contradictorily perceive a lower level of social support due to their escalating inner needs (Miller et al. 2015, Pisula \& Lukowska 2012).

The mean scores of the adolescents surveyed in this study in psychological capital and the dimensions are between 3.7 and 3.9. Fan et al. (2018a) investigated 646 rural Chinese adolescents, finding that their scores in the dimensions of psychological capital are between 3.4 and 4.1. These scores differ from the results of this paper. The research tools used in this paper are consistent with those in the study by Fan et al. (2018a), and the differences between the results may be related to the heterogeneity of the samples. The sample surveyed in this study is ordinary adolescents, while Fan et al. (2018a) focused on left-behind children in rural areas Left-behind children refer to children who are left alone at home, live with other relatives, or live alone because their parents work in another place. These children have certain peculiarities, and their living environment and psychological development status are different from those of ordinary children (Fan et al. 2016).

Furthermore, the loneliness' mean score of the adolescents surveyed in this study is roughly 15 points, which is consistent with the finding of Xu et al. (2017). In their study, the mean score of adolescents in loneliness is about 16 points. It can be seen from the mean value that adolescents' loneliness is at a medium level. Nonetheless, a medium level does not mean a low sense of loneliness among adolescents, because the form of self-report may lead to hidden behaviors for avoiding social appreciative effects (Lodder et al. 2016).

\section{Analysis of correlation between perceived social support, psychological capital and loneliness among adolescents}

According to the results of this paper, a significant positive correlation exists between perceived social support and each dimension of their psychological capital. That is, more perceived social support result in a 
higher level of psychological capital among adolescents. This paper is consistent with the findings of a survey on 320 individuals by Liu et al. (2013). In their research, perceived social support and psychological capital are found to be negatively correlated with negative mental health indicators such as depression and anxiety, and perceived social support is positively correlated to psychological capital. Researchers of the theory of psychological capital believed that in addition to trait psychological capital, psychological capital has a statelike characteristic, meaning that psychological capital has a dynamic nature (Luthans et al. 2006). Meanwhile, studies have show that perceived social support has a positive effect on different aspects of psychological capital such as self-efficacy, optimism, hope, and resilience. For example, Foote et al. (1990) found in a strong positive correlation between perceived social support and hope, self-efficacy, and self-esteem. Schönfeld et al. (2017), found a positive correlation between initial self-efficacy and self-reported perceived social support one year later. In their study of traumatic stress therapy, it was reported that perceived social support has a positive effect on optimism. Luthans et al. (2008) verified directly that a supportive environment has a positive effect on psychological capital, basically consistent with the results obtained by Wang et al. (2018).

The results of this paper show the existence of a significant negative correlation between each dimension of perceived social support and loneliness, which means adolescents perceiving more social support would report a lower level of loneliness. To a certain extent, this result proves that perceived social support is an important factor affecting loneliness among adolescents. This conclusion is consistent with that obtained by HoltLunstad et al. (2015). The family is the initial environment for the socialization of adolescents and parents are the most important source of perceived social support in early adolescence (An 2010). The development of adolescents' emotions and social relationships is related directly to their families. Therefore, when the emotional link between the parents and the adolescent from the family is reduced, or when the parents' emotional care for the adolescent is reduced, the adolescent's perceived social support from the family is reduced, which may lead to the adolescent's rising experience of loneliness, especially in the early stages. Furthermore, with the arrival of puberty, peer relationships begin to play an increasingly important role in the growth and development of adolescents. A large part of the perceived social support adolescents need during this period is closely related to their friendships with friends and peers (Lodder et al. 2016). Loyalty, mutual help and mutual support are often seen as signs of effective perception of social support among adolescents because good peers or friendships play an important role in at least three aspects: First, intimate peers are reliable listeners for adolescents who suffer from various emo- tional or behavioral problems. Appropriate confession allows adolescents to vent negative emotions in a timely manner and promote their mental health considerably (Li et al. 2017). Second, intimate peers are models for adolescents to learn and imitate actively. By observing their peers' styles of coping with positive or negative events, adolescents can shape their own coping styles to some extent; third, peers sometimes play a warning role, and adolescents can learn from their peers the negative consequences of bad behaviors, thereby reducing the likelihood of problematic behaviors.

The results of this study also show a significant negative correlation exists between adolescents' psychological capital and loneliness, and between each dimension of these two. In other words, a higher level of psychological capital brings less experience of loneliness among adolescents. This finding is consistent with the result of Fan et al. (2018b) who investigated 971 rural children, including left-behind and non-left-behind children, and found a negative correlation between psychological capital and loneliness, between both leftbehind children and non-left-behind children. To some extent, these findings indicate psychological capital is a positive protective factor for adolescents' social adaptation (Novak \& Kawachi 2015). The stress buffer hypothesis believes individuals with higher psychological capital are more likely to regard stress as a challenge rather than a hindrance or threat in face of life stress, thus giving stress positive meaning and promoting positive inner motivation. They are also more inclined to adopt a positive and mature coping style, thereby reducing negative emotional experience in the face of stressful events (Fan et al. 2018b). Specifically, adolescents with higher psychological capital would take positive coping measures, resulting in a decrease in their negative emotions such as loneliness. On the contrary, individuals with lower psychological capital pay more attention to negative stimuli in the environment, and the emotional response would be the choice of coping style (Ding et al. 2015). It was shown in previous studies that higher emotional coping cannot improve the individual's positive emotional experience but may enhance their negative emotional experience (Zou et al. 2015).

\section{Mediating role of psychological capital in the relationship between perceived social support and loneliness}

The results of this paper show that perceived social support not only predicts loneliness among adolescents significantly, but also affects their loneliness indirectly through the partial mediating effect of psychological capital. This finding is consistent with previous research results. For example, in a survey of 381 college students, Li et al. (2014) found that psychological capital plays a mediating role in the relationship between perceived social support and subjective well- 
being. Although a general consensus has been reached on the positive effect of perceived social support on adolescents' mental health, few studies have focused on how perceived social support affects adolescents' mental health, that is, the mediating mechanism for the relationship between perceived social support and adolescents' mental health (Yu \& Liang 2017). Perceived social support promotes positive emotional experiences in adolescents by affecting their emotions, cognition, and behaviors (Lee et al. 2018). However, perceived social support is in a somewhat opposite position to social isolation or rejection and pressure, and the results of Fan et al. (2017) are basically the same. In their research, psychological capital plays a partial mediating role in the relationship between children's life stress and loneliness.

The mediating role of psychological capital in the relationship between perceived social support and loneliness can be explained separately from the perspective of conservation of resource theory and stress process theory. First, conservation of resource theory posits that people always pursue the minimum loss and maximum maintenance of internal psychological resources (Chen \& Wu 2017). Those positive events aid individuals not only to preserve existing resources but also to increase psychological resources. When sufficient psychological resources exist, individuals would experience positive emotions. In the face of stressful life events, individuals have to consume their internal psychological resources. If the sustained consumption is larger than the acquisition of psychological resources, then individuals may fall into the loss of confidence, ultimately affecting their social adaptation. This loss of confidence will result in various emotional problems including loneliness. Second, stress process theory points out that stress can affect individuals' emotional health directly or affect their emotions directly by depleting certain psychological resources, such as control and self-efficacy. According to the stress buffer hypothesis, positive psychological capital helps individuals deal with life events actively, both under high stress and low stress, thereby reducing negative emotional experiences. In some studies that follow the research paradigm of "stress-resource-adaptation", it was also found that psychological capital plays a mediating role between stress and mental health (Kwag et al. 2011).

\section{CONCLUSIONS}

The present paper found the following: (1) Significant correlations exist between perceived social support, psychological capital, and loneliness of adolescents. A significant negative correlation also exists between perceived social support and loneliness, as well as between significant positive correlation between perceived social support and psychological capital and a significant negative correlation between psychological capital and loneliness. (2) psychological capital plays a partial mediating role between perceived social support and loneliness among adolescents. his paper provides a theoretical basis for making targeted and effective interventions for adolescents' loneliness. These findings could have considerable significance in alleviating their loneliness, enhancing their perceived social support, and promoting their healthy development. Schools and teachers should pay attention to adolescents' loneliness, ensure timely detection and make early interventions to prevent and alleviate effectively the symptoms of loneliness. School administrators should organize more group activities, endeavor to improve adolescents' positive psychological qualities and other psychological capital, and encourage adolescents to participate actively in such activities. Teachers should also participate in group activities, give students encouragement and care as much as possible, and improve their level of perceived social support. Parents should adopt a moderate and equal education style to strengthen adolescents' perception of social support and to alleviate their feelings of loneliness.

\section{Acknowledgements: None.}

\section{Conflict of interest: None to declare.}

\section{Contribution of individual authors:}

Yujia Ren \& Binbin Ji designed the study.

Yujia Ren wrote the manuscript, all authors contributed to and have approved the final manuscript.

\section{References}

1. An PP: Parent-child Relationship and the Socialization of Young People. Social Sciences Journal of Colleges of Shanxi 2010; 22:12-14

2. Bian M, Leung L: Linking loneliness, shyness, smartphone addiction symptoms, and patterns of smartphone use to social capital. Social Science Computer Review 2015; 33:61-79

3. Carmona-Halty $M$, Schaufeli WB, Salanova M: Good Relationships, Good Performance: The Mediating Role of Psychological Capital-A Three Waves Study among Students. Frontiers in Psychology 2019; 10:306

4. Cavanaugh AM, Buehler C: Adolescent loneliness and social anxiety: The role of multiple sources of support. Journal of social and personal relationships 2016; 33:149-170

5. Chen JA, Wu XM: Can "Mixed-sex Dyad" in Workplace Stimulate Individual's Tireless Working Experience? Based on the Conservation of Resources Theory. Luojia Management Review 2017; 15:49-62

6. Ding Y, Yang Y, Yang X, Zhang T, Qiu X, He X, et al.: The mediating role of coping style in the relationship between psychological capital and burnout among Chinese nurses. PloS one 2015; 10:e0122128 
7. Fan XH, Fang XY, Chen FJ, Huang YS, Zhang SY: The Development of Psychological Capital Scale for Parentabsent Children in Rural Areas of China. Chinese Journal of Clinical Psychology 2015; 23:1-6

8. Fan XH, Jian JP, Chen FJ, Yu MJ, Zhou Y, Chen Q: Family Adversity and Psychological Adaptation among the Left-behind Children. Chinese Journal of Clinical Psychology 2018b; 26:353-357

9. Fan XH, Lu X, Chen FJ: The Structure and Impact of Psychological Capital of Left-behind Children in Rural Areas: A Qualitative Study. Hunan Social Sciences 2016; 4:67-70

10. Fan XH, Yu S, Peng J, Fang XY: The Relationship between Perceived Life Stress, Loneliness and General WellBeing among the Left-behind Rural Children: Psychological Capital as a Mediator and Moderator. Journal of Psychological Science 2017; 40:388-394

11. Fan XH, Zhou L, He Q, She LZ, Zhu D, Meng H: Effect of Psychological Capital on Academic Achievement on Leftbehind Children in Rural China: a Moderated Mediating Effect. Chinese Journal of Clinical Psychology 2018a; 26:135-140

12. Foote AW, Piazza D, Holcombe J, Paul P, Daffin P: Hope, self-esteem and social support in persons with multiple sclerosis. The Journal of neuroscience nursing 1990; 22:155-159

13. He F, Guan H, Kong Y, Cao R \& Peng J: Some individual differences influencing the propensity to happiness: Insights from behavioral economics. Social Indicators Research 2014; 119:897-908

14. Heinrich LM, Gullone E: The clinical significance of loneliness: A literature review. Clinical psychology review 2006; 26:695-718

15. Holt-Lunstad J, Smith TB, Baker M, Harris $T$ \& Stephenson D: Loneliness and social isolation as risk factors for mortality: a meta-analytic review. Perspectives on Psychological Science 2015;10:227-237

16. Jaremka LM, Fagundes CP, Glaser R, Bennett JM, Malarkey WB \& Kiecolt-Glaser JK: Loneliness predicts pain, depression, and fatigue: understanding the role of immune dysregulation. Psychoneuroendocrinology 2013, 38:1310-1317

17. Kwag KH, Martin P, Russell D, Franke W, Kohut M: The impact of perceived stress, social support, and homebased physical activity on mental health among older adults. The International Journal of Aging and Human Development 2011; 72:137-154

18. Laird Y, Fawkner S, Kelly P, McNamee L, Niven A: The role of social support on physical activity behaviour in adolescent girls: a systematic review and meta-analysis. International Journal of Behavioral Nutrition and Physical Activity 2016; 13:79

19. Lee S, Chung JE, Park N: Network environments and well-being: an examination of personal network structure, social capital, and perceived social support. Health communication 2018; 33:22-31

20. Li B, Ma H, Guo Y, Xu F, Yu F, Zhou Z: Positive psychological capital: $A$ new approach to social support and subjective well-being. Social Behavior and Personality 2014; 42:135-144

21. Li ML, Jiang X, Ren YJ: Mediator effects of positive emotions on social support and depression among adolescents suffering from mobile phone addiction. Psychiatr Danub 2017; 29:207-213
22. Liu L, Gou Z, Zuo J: Social support mediates loneliness and depression in elderly people. Journal of health psychology 2016; 21:750-758

23. Liu L, Pang R, Sun W, Wu M, Qu P, Lu C, et al.: Functional social support, psychological capital, and depressive and anxiety symptoms among people living with HIV/AIDS employed full-time. BMC psychiatry 2013; 13:324

24. Lodder GMA, Goossens L, Scholte RHJ, Engels RCME, Verhagen M: Adolescent loneliness and social skills: Agreement and discrepancies between self-, meta-, and peer-evaluations. Journal of youth and adolescence 2016; 45:2406-2416

25. Luthans F, Avey JB, Avolio BJ, Norman SM, Combs GM: Psychological capital development: toward a micro-intervention. Journal of Organizational Behavior: The International Journal of Industrial, Occupational and Organizational Psychology and Behavior 2006; 27:387-393

26. Luthans F, Avey JB, Patera JL: Experimental analysis of a web-based training intervention to develop positive psychological capital. Academy of Management Learning \& Education 2008; 7:209-221

27. Miller AB, Esposito-Smythers C, Leichtweis RN: Role of social support in adolescent suicidal ideation and suicide attempts. Journal of Adolescent Health 2015; 56:286-292

28. Mosher CE, Lepore SJ, Wu L, Austin J, Valdimarsdottir H, Rowley $S$, et al.: Social correlates of distress following hematopoietic stem cell transplantation: Exploring the role of loneliness and cognitive processing. Journal of health psychology 2012; 17:1022-1032

29. Novak D, Kawachi I: Influence of different domains of social capital on psychological distress among Croatian high school students. International journal of mental health systems 2015; 9:1-7

30. Park J, Kitayama S, Karasawa M, Curhan K, Markus HR, Kawakami N, et al.: Clarifying the links between social support and health: Culture, stress, and neuroticism matter. Journal of health psychology 2013; 18:226-235

31. Peterson SJ, Luthans F, Avolio BJ, Walumbwa FO \& Zhang Z: Psychological capital and employee performance: a latent growth modeling approach. Personnel Psychology 2011; 64:427-450

32. Pisula E, Lukowska E: Perception of social relationships with classmates and social support in adolescents with asperger syndrome attending mainstream schools in Poland. School Psychology International 2012; 33:185-206

33. Schönfeld P, Preusser F, Margraf J: Costs and benefits of self-efficacy: differences of the stress response and clinical implications. Neuroscience \& Biobehavioral Reviews 2017; 75:40-52

34. Wang JK, Chen J, Hao XJ, Zhang P: Effect of learning burnout on life satisfaction: Mediating effect of psychological capital and perceived social support in college students. Chinese Mental Health Journal 2018; 32:85-89

35. Wen ZL, Zhang L, Huo JT, Liu HY: Testing and Application of the Mediating Effects. Acta Psychologica Sinica 2004; 36:614-620

36. Xu HH, Sun XJ, Zhou ZK, Niu GF, Lian SL: True SelfExpression on Social Network Sites and Loneliness: Mediating of Self-Concept Clarity. Chinese Journal of Clinical Psychology 2017; 25:138-141

37. Yan BB, Zheng X: Researches into Relations among Social-support, Self-esteem and Subjective Well-being of College Students. Psychological Development and Education 2006; 22:60-64 
38. Ye BJ, Hu XY, Yang Q, Hu ZQ: The Effect Mechanism of Perceived Social Support, Coping Efficacy and Stressful Life Events on Adolescents' Academic Achievement. Journal of Psychological Science 2014; 37:342-348

39. Yu L, Liang J: Perceived Social Support and Internalizing Problem among Adolescents: The Moderating Effect of Self-esteem. China Journal of Health Psychology 2017; $25: 227-230$

40. Zhang F, Wang JN, Jin TL: Perceived Social Support on Adolescent Depression: Mediating Role of Self-esteem and Regulation Role of Gender. Psychology: Techniques and Applications 2017; 5:665-671
41. Zhou L, Li Z, Hu M, Xiao SY: Reliability and validity of ULS-8 loneliness scale in elderly samples in a rural community. Journal of Central South University (Medical Science) 2012; 37:1124-1128

42. Zimet GD, Powell SS, Farley GK, Werkman S, \& Berkoff KA: Psychometric characteristics of the multidimensional scale of perceived social support. Journal of Personality Assessment 1990; 55:610-617

43. Zou H, Liu Y, Zhang WJ, Jiang S, Zhou H, Yu YB: Adolescents' Social Adjustment: A Conceptual model, Assessment and Multiple Protective and Risk Factors. Psychological Development and Education 2015; 31:29-36

Correspondence:

Associate Professor Yujia Ren, MD

Physical Education Institute, Hunan First Normal University

The Third Fenglin Road, 410205 Changsha City, Hunan Province, China

E-mail: renyujia@163.com 